\title{
Accurate and Efficient Fault Simulation of Realistic CMOS Network Breaks
}

\author{
Haluk Konuk F. Joel Ferguson Tracy Larrabee \\ Computer Engineering Board of Studies, University of California at Santa Cruz
}

\begin{abstract}
We present a new fault simulation algorithm for realistic break faults in the p-networks and n-networks of static CMOS cells. We show that Miller effects can invalidate a test just as charge sharing can, and we present a new charge-based approach that efficiently and accurately predicts the worst case effects of Miller capacitances and charge sharing together. Results on running our fault simulator on ISCAS85 benchmark circuits are provided.
\end{abstract}

\section{INTRODUCTION}

Defects that occur during the IC manufacturing process can be categorized into three classes according to Hawkins et al. [7]. These classes are bridge, open circuit, and parametric defects. Open circuit defects cause breaks in the conducting materials in the layout, and contacts are particularly susceptible to such breaks. Breaks can be divided into two categories: those that physically disconnect one or more transistor gates from their drivers, and those that disconnect transistors from each other in the p-network or n-network of a CMOS cell [12]. We define a network break as a break fault in the p-network or in the n-network of a cell that breaks one or more transistor paths between the cell output and Vdd or GND. A transistor path is a sequence of transistors physically connected through their drain and source terminals. Note that transistor stuckopen faults form a subset of network break faults. Renovell and Cambon [16], and Champac et al. [1] showed that a transistor stuck-open test set can detect some of the breaks that create floating transistor gates. So, a network break test set is useful not only for detecting network breaks but also other breaks that cause floating transistor gates.

Detection of a network break with voltage measurements requires a two-vector test. Reddy et al. [15] showed that transient paths to Vdd or GND can invalidate a two-vector test in transistor stuck-open testing, and Barzilai et al. showed that charge sharing between the internal nodes of the faulty cell and the high impedance faulty cell output can also invalidate a test. Lee and Breuer [11] proposed a

Work supported by SRC contract 94-DJ-315 and NSF grants MIP9158490 and MIP-9158491.

32nd ACM/IEEE Design Automation Conference ${ }^{\circledR}$

Permission to copy without fee all or part of this material is granted, provided that the copies are not made or distributed for direct commercial advantage, the ACM copyright notice and the title of the publication and its date appear, and notice is given that copying is by permission of the Association for Computing Machinery. To copy otherwise, or to republish, requires a fee and/or specific permission. scheme for handling charge sharing in transistor stuck-open fault testing using both IDDQ and voltage measurements, but the resulting test sizes might be prohibitive for IDDQ testing. Barzilai et al. [5] described a fault simulator for transistor stuck-open and stuck-on faults. For handling charge sharing, they partitioned all the nodes in every cell into two classes. Nodes in the first class were assumed to have small enough capacitances so that they could be ignored. If a node in the second class can share charge with the floating cell output, then the test is declared invalidated. Di and Jess [3] developed a fault simulator for network breaks, but they ignored static hazards, and their detecting conditions considered charge sharing only with the nodes on the broken paths. Favalli et al. [6] proposed a set of detection conditions for network breaks, but they considered neither transient paths to Vdd or GND, nor charge sharing.

In this paper we present a new charge-based fault simulation algorithm for network breaks that takes into account the transient paths to Vdd or GND, charge sharing, Miller feedback effect, and Miller feedthrough effect. We demonstrate in Section 2 that Miller capacitances can invalidate a two-vector test for a network break just as charge sharing can. To the best of our knowledge, there is no other published work that considers the Miller effect on test invalidation in network break or transistor stuck-open fault simulation.

Because we have a charge-based approach, the nonlinearity of Miller and $\mathrm{p}-\mathrm{n}$ junction capacitances are accurately modeled compared to previous capacitance-based approaches. In Sections 2.1 and 2.2, we show that a Miller capacitance and a $\mathrm{p}-\mathrm{n}$ junction capacitance can vary by more than a factor of five and a factor of two, respectively.

We use only six voltage levels to compute the worst case charge differences, as described in Section 3.2, so the charge equations can be precomputed into a look-up table. Section 4 shows that our CPU times are very competitive with previous less accurate fault simulation methods.

\section{DETECTION OF NETWORK BREAKS}

To guarantee the detection of a network break with voltage measurements, a two-vector test is necessary. Without loss of generality, let us assume that the break is in the p-network. Then, the first vector should initialize the cell output to GND, and the second vector should activate only the broken paths in the p-network and no other path. Activating a path means applying $\mathrm{ON}$ voltages to the gates of all the transistors on the path. The second vector will make the faulty cell output high impedance with GND as its initial 
voltage. If the faulty cell output keeps its logic 0 value until the circuit outputs are sampled, and the second vector is a test for the cell output stuck-at-0 fault, then the network break will be detected. If certain mechanisms, which can raise the high-impedance cell output voltage, are not taken into account, a two-vector sequence may be incorrectly classified as a test for the break.

Two mechanisms that may invalidate a test, transient paths to Vdd or GND and charge sharing, have been studied by many researchers $[15,9,20,2,5,11,3]$. In this paper, we show that the gate-drain or the gate-source capacitances of the CMOS transistors can modify the voltage of the faulty cell output when it is at high impedance. We refer to these capacitances as Miller feedthrough [14] when they are inside the faulty cell, and as Miller feedback [14] when they are inside the fanout cells of the faulty cell.

We now introduce some terminology that will be used in the rest of the paper. Let time-frame $\mathbf{1}$ or TF-1 denote the time interval beginning with the application of the first vector and ending with the application of the second vector, and let time-frame $\mathbf{2}$ or TF-2 begin with the application of the second vector and end with the sampling of the circuit outputs. We assume that all the signals in the circuit will be stable by the end of time-frames 1 and 2 .

We use an eleven-value logic algebra for the logic values of wires in the two time frames. Let $a b$ denote one of the nine values of our logic algebra, where $a, b \in\{0,1, X\}$, and $a$ and $b$ are the final values of a wire in TF- 1 and TF-2, respectively. Thus, 00 on wire $l$ means that the final value of $l$ is 0 in both time frames. Due to multiple paths from circuit inputs to line $l$, the value on $l$ may temporarily change to 1 and change back to 0 again, which is called a static hazard. As the other two values of our eleven-value logic algebra, we use S0 to represent a 00 with no static hazard, and $\mathbf{S 1}$ to represent a 11 with no static hazard, and refer to them as stable 0 and stable 1 [19], respectively.

In this paper, our emphasis is on how Miller feedback and feedthrough effects, and charge sharing can invalidate a test. We use the circuit in Figure 1 to demonstrate these test invalidation mechanisms. The cell on the left in Figure 1 with a p-network break in it is an OAI31, and the cell on the right is a NOR gate, both from the MCNC cell library. We used HSPICE to simulate this circuit. We used level 13 (the BSIM model) in HSPICE, because this model guarantees charge conservation. We obtained the BSIM model parameters from MOSIS for the $1.2 \mu$ Orbit $\mathrm{n}$-well fabrication process. The $35 \mathrm{fF}$ capacitance shown in Figure 1 is used to model a metal- 1 wire that is around $160 \mu$ long in this $1.2 \mu$ process.

\section{$2.1 \quad$ Miller Feedback Effect}

We now show that the voltage changes on the drain/source terminals of a transistor can significantly change the voltage of its floating gate (Miller feedback effect). We want to emphasize that a Miller feedback capacitance is not only due to the overlap between the gate and diffusion regions of a transistor, but it is also due to the charge stored in the channel region, and it can go up to half of the

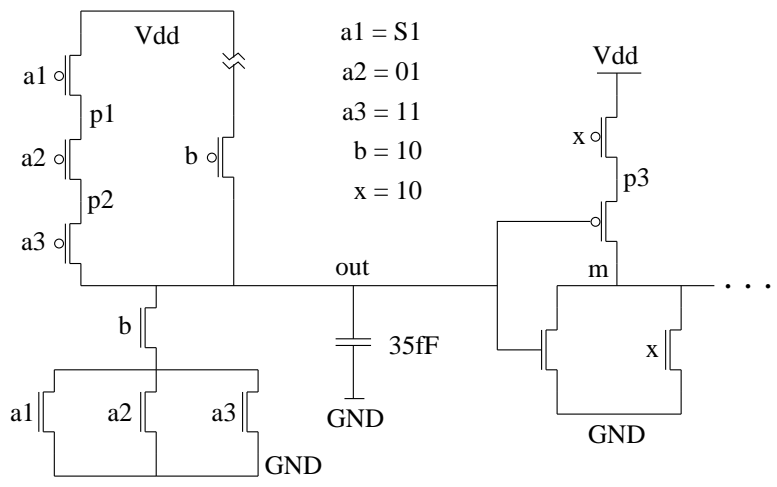

Figure 1: A circuit to demonstrate test invalidation

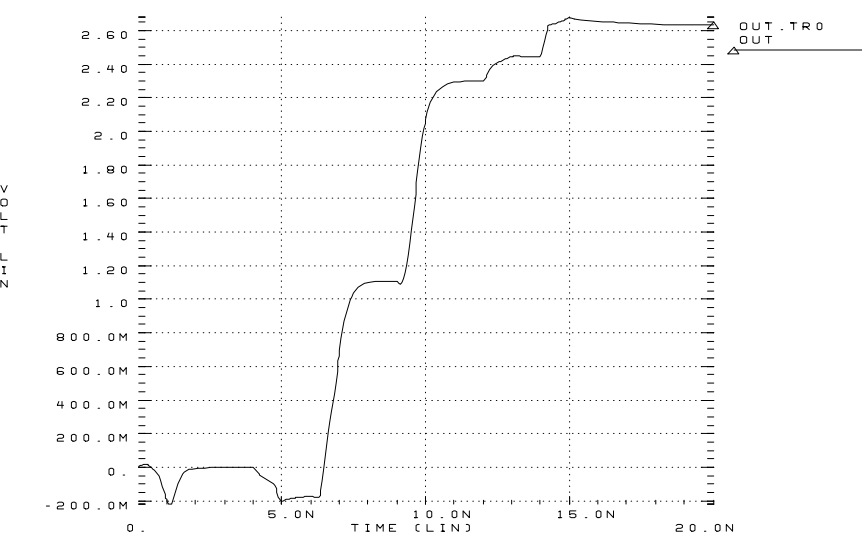

Figure 2: Test invalidation by Miller feedback, charge sharing, and Miller feedthrough

total gate capacitance when the transistor is on. For the pMOS transistor connected to out in the NOR gate in Figure 1, the Miller feedback capacitance changes from 4.1fF to $20.8 \mathrm{fF}$ according to HSPICE when the transistor gate voltage changes from $5 \mathrm{~V}$ to $0 \mathrm{~V}$ with drain and source voltages held at $5 \mathrm{~V}$.

Consider the proposed test shown in Figure 1. Table 1 shows the simulated behavior of all the cell inputs in TF-2 and in part of TF-1. We assume that the circuit in Figure 1 is embedded in a larger circuit, and the cell inputs are not the primary inputs. The first transition in TF-2 happens at line $b$ making the OAI31 output floating with a slightly negative initial voltage as shown in Figure 2. The next transition is at $x$ between $6 \mathrm{~ns}$ and $7 \mathrm{~ns}$. Just before this transition, the NOR output $m$ was at $0 \mathrm{~V}$, and the internal node $p 3$ in the NOR gate was at around $1.2 \mathrm{~V}$, which is about the minimum voltage an internal $\mathrm{p}$-diffusion node can acquire in the process we used. After $x$ becomes $0 \mathrm{~V}$ turning on the pMOS transistor it is connected to, $p 3$ and $m$ both rise to around $5 \mathrm{~V}$. These rising transitions on $p 3$ and $m$ raise the out voltage due to Miller feedback to $1.1 \mathrm{~V}$ from $6 \mathrm{~ns}$ to $9 \mathrm{~ns}$ as shown in Figure 2.

In TF- $1 x$ is $0 \mathrm{~V}$ to first charge up $p 3$ to $5 \mathrm{~V}$, and then let it drain down to $1.2 \mathrm{~V}$ when $b$ becomes high impedance. 


\begin{tabular}{|c|c|c|c|c|c|c|c|c|c|c|c|c|}
\hline & \multirow{2}{*}{\multicolumn{2}{|c|}{$\begin{array}{c}\text { Part of TF-1 } \\
\text { initializing } \\
p 1, p 2, p 3\end{array}$}} & \multicolumn{10}{|c|}{ Time Frame 2} \\
\hline & & & \multicolumn{2}{|c|}{$\begin{array}{c}\text { out starts } \\
\text { floating }\end{array}$} & \multicolumn{2}{|c|}{$\begin{array}{c}\text { Miller } \\
\text { feedback }\end{array}$} & \multicolumn{2}{|c|}{$\begin{array}{c}\text { charge } \\
\text { sharing }\end{array}$} & \multicolumn{4}{|c|}{$\begin{array}{c}\text { Miller } \\
\text { feedthrough }\end{array}$} \\
\hline & Ons & 1ns & $4 n s$ & $5 \mathrm{~ns}$ & $6 \mathrm{~ns}$ & $7 \mathrm{~ns}$ & $9 \mathrm{~ns}$ & $10 \mathrm{~ns}$ & $12 \mathrm{~ns}$ & $13 n s$ & $14 \mathrm{~ns}$ & $15 \mathrm{~ns}$ \\
\hline$x$ & OV & $5 \mathrm{~V}$ & $5 \mathrm{~V}$ & $5 \mathrm{~V}$ & $5 \mathrm{~V}$ & $0 \mathrm{~V}$ & $0 \mathrm{~V}$ & $0 \mathrm{~V}$ & $0 \mathrm{~V}$ & $0 \mathrm{~V}$ & $0 \mathrm{~V}$ & $0 \mathrm{~V}$ \\
\hline$a 1$ & $0 \mathrm{~V}$ & $5 \mathrm{~V}$ & $5 \mathrm{~V}$ & $5 \mathrm{~V}$ & $5 \mathrm{~V}$ & $5 \mathrm{~V}$ & $5 \mathrm{~V}$ & $5 \mathrm{~V}$ & $5 \mathrm{~V}$ & $5 \mathrm{~V}$ & $5 \mathrm{~V}$ & $5 \mathrm{~V}$ \\
\hline$a 2$ & $0 \mathrm{~V}$ & $0 \mathrm{~V}$ & $0 \mathrm{~V}$ & $0 \mathrm{~V}$ & $0 \mathrm{~V}$ & $0 \mathrm{~V}$ & $0 \mathrm{~V}$ & $0 \mathrm{~V}$ & OV & $5 \mathrm{~V}$ & $5 \mathrm{~V}$ & $5 \mathrm{~V}$ \\
\hline$a 3$ & $5 \mathrm{~V}$ & $5 \mathrm{~V}$ & $5 \mathrm{~V}$ & $5 \mathrm{~V}$ & $5 \mathrm{~V}$ & $5 \mathrm{~V}$ & $5 \mathrm{~V}$ & 0V & $0 \mathrm{~V}$ & $0 \mathrm{~V}$ & OV & $5 \mathrm{~V}$ \\
\hline$b$ & $5 \mathrm{~V}$ & $5 \mathrm{~V}$ & $5 \mathrm{~V}$ & 0V & $0 \mathrm{~V}$ & $0 \mathrm{~V}$ & $0 \mathrm{~V}$ & $0 \mathrm{~V}$ & $0 \mathrm{~V}$ & $0 \mathrm{~V}$ & $0 \mathrm{~V}$ & $0 \mathrm{~V}$ \\
\hline
\end{tabular}

Table 1: The simulated behavior of the cell input signals in Figure 1

\subsection{Charge Sharing}

We assume that the next transition in TF-2 is at line $a 3$ between $9 \mathrm{~ns}$ and $10 \mathrm{~ns}$ due to a glitch. Now, out is connected to internal nodes $p 1$ and $p 2$ in the OAI31 cell. Since $p 1$ and $p 2$ were initialized to $5 \mathrm{~V}$ during TF-1 by starting $a 1$ at $0 \mathrm{~V}$, charge transfer from $p 1$ and $p 2$ to out raises the out voltage to $2.3 \mathrm{~V}$ from $9 \mathrm{~ns}$ to $12 \mathrm{~ns}$ as shown in Figure 2 . The p$\mathrm{n}$ junction capacitance of node $p 2$ changes from $26.7 \mathrm{fF}$ to $14.9 \mathrm{fF}$ when the voltage at $p 2$ changes from $5 \mathrm{~V}$ to $2.3 \mathrm{~V}$. When the voltage at $p 2$ drops to $1 \mathrm{~V}$, its capacitance drops to $13.2 \mathrm{fF}$.

\section{$2.3 \quad$ Miller Feedthrough Effect}

The next event is a rising transition at line $a 2$ between $12 \mathrm{~ns}$ and $13 \mathrm{~ns}$. Due to the gate-drain and gate-source (Miller feedthrough) capacitances of the pMOS transistor $a 2$ is connected to, this transition raises the voltages on $p 1$ and $p 2$. The voltage increase on $p 2$ enables additional charge transfer from $p 2$ to out between $12 \mathrm{~ns}$ and $14 \mathrm{~ns}$. The final event is a rising transition at line $a 3$ between $14 \mathrm{~ns}$ and $15 \mathrm{~ns}$, which bumps up the out voltage to its final value of $2.63 \mathrm{~V}$., which is interpreted as logic 1 by the NOR gate. Thus, the test is invalidated.

\section{THE FAULT SIMULATION ALGORITHM}

Our fault simulation algorithm declares a two-vector sequence a test for a network break if the sequence cannot be invalidated by transient paths to Vdd or GND, Miller feedback and feedthrough effects, and charge sharing. We first perform a gate level simulation using our eleven-value logic algebra. We assume that if a circuit input has the same logic value in time frames 1 and 2, then that input has no static hazard, that is, it is glitch-free. For an AND gate to have an $\mathrm{S} 0$ value at its output, at least one of its inputs must be $\mathrm{S} 0$, and to have an S1 at its output, all of its inputs must be $\mathrm{S} 1$. An OR gate is processed similarly.

In order to guarantee that there will not be any transient path to $V$ dd for a p-network break, all the paths from the faulty cell output to $\mathrm{Vdd}$ in the p-network must have at least one transistor with $\mathrm{S} 1$ value at its gate. This is both a necessary and sufficient condition. Similarly, in order to guarantee no transient path to GND for an n-network break, all the paths from the faulty cell output to GND must have at least one transistor with $\mathrm{S} 0$ value at its gate.

In order to guarantee that a test will not be invalidated by Miller effects and charge sharing, our fault simulator uses a charge-based approach that computes the worst case charge transfer from Miller and $\mathrm{p}-\mathrm{n}$ junction capacitances to the floating faulty cell output and vice versa. This approach is described next.

\subsection{A Charge-Based Approach}

When a test for a network break is applied, the faulty cell output starts floating at some point during TF-2, and stays floating in the rest of TF-2. We refer to this time period as the floating period. We assume that TF-2 is short enough so that the transistor leakage currents can be ignored. During the floating period, voltage changes at the gates of the transistors in the faulty cell can displace charge from, or bring in more charge to, the drain and source terminals (Miller feedthrough effect); the output may be connected to some internal nodes in the faulty cell resulting in charge sharing; and voltage changes at the internal nodes of the fanout cells can displace charge from, or bring in more charge to, the gate terminals of the transistors fed by the floating output (Miller feedback effect). Assuming constant values for the Miller and $\mathrm{p}-\mathrm{n}$ junction capacitances would be too pessimistic or too optimistic, because these capacitances can vary up to a factor of five as shown in Section 2.1. So, our approach is based on computing the worst case changes in electrical charge as a function of the worst case voltage changes at the inputs of the faulty cell and its fanout cells.

Let us now identify the components of the charge stored at the faulty cell output $O$, and at a faulty cell internal node. Let $\mathbf{I}$ denote the set formed by the faulty cell internal nodes that might be connected to $O$ during the floating period, and FCN $=I \cup\{O\}$ where $F C N$ stands for the set of $F$ aulty Cell Nodes. The following two components exist for the charge stored on any faulty cell node fcn $\in F C N$.

1. Each transistor drain or source terminal ds connected to $f \mathrm{cn}$ stores charge in the intrinsic, or channel, area of the transistor $\mathbf{t}$ when $t$ is on [18]. Some charge is also stored on $d s$ due to the gate overlap capacitance. We denote the charge on $d s$ of $t$ as $\mathbf{Q}_{\mathbf{d s}, \mathbf{t}}$.

2. Charge is stored in the $\mathrm{p}-\mathrm{n}$ junction between $\mathrm{fcn}$ and the bulk of the transistor, which we denote as $\mathrm{Q}_{\mathbf{p}-\mathbf{n}, \mathbf{f} \mathbf{c n} \text {. }}$

The following two charge components exist only for the faulty cell output $O$ :

3. Charge is stored on the gate of each fanout transistor $\mathbf{f}$ connected to $O$. We denote this charge as $\mathbf{Q}_{\mathrm{g}, \mathbf{f}}$.

4. Charge is stored on the metal wire that connects the faulty cell to its fanout cells, due to the linear capacitance to GND and to nearby wires. In this paper, we 
ignore the interwire capacitances, and consider only the capacitance to GND, which we refer to as the wiring capacitance. We denote this charge as $\mathbf{Q}_{\text {wiring. }}$.

Let us assume for now that the total charge stored at the nodes in $F C N$ at $t_{\text {init }}$ is the same as the charge stored at $t_{\text {final }}$, where $\mathbf{t}_{\text {init }}$ denotes the beginning of the floating period, and $\mathbf{t}_{\text {final }}$ denotes the end of the floating period, which is also the end of TF-2. So, we will assume that charge is conserved during the floating period. We are interested in the worst case charge difference on the wiring capacitance $\mathrm{C}_{\mathrm{O} \text {,wiring, because this charge difference } \Delta Q_{\text {wiring }} \text { will }}$ give us the worst case voltage change on $O$. Because of charge conservation, any charge difference on the wiring capacitance, which represents only component 4 of the charge stored on $O$, must come from the charge differences on the remaining three charge components of $O$ and from the charge differences in the nodes of $I$. Therefore, $\Delta Q_{\text {wiring }}$ can be expressed as follows.

$$
\begin{gathered}
\Delta Q_{\text {wiring }}=-\left(\sum_{f c n \in F C N} \Delta Q_{f c n}+\sum_{f \in F} \Delta Q_{g, f}\right) \\
\Delta Q_{f c n}=\Delta Q_{p-n, f c n}+\sum_{t \in T_{f c n}} \Delta Q_{d s, t}
\end{gathered}
$$

where $F$ is the set of transistors whose gates are connected to $O$, and $T_{f c n}$ is the set of transistors whose drain or source terminals are connected to $f \mathrm{cn}$. The worst case charge differences are determined by the worst case voltage differences from $t_{\text {init }}$ to $t_{\text {final }}$. Section 3.2 describes how we obtain these worst case voltages. In Equation 3.2, the $\Delta Q_{p-n, f e n}$ term is for charge sharing between nodes $f c n$ and $O$, and the summation term is for the Miller feedthrough effect of the transistors in $T_{f e n}$. In Equation 3.1, the second summation term is for the Miller feedback effect.

Let Lo_th and L1_th denote the maximum voltage that is still a logic 0 and the minimum voltage that is still a logic 1 , respectively. If the faulty cell output $O$ is initialized to $0 \mathrm{~V}$ in TF-1, implying a p-network break, then we assume that $O$ will reach $\mathrm{L} 0 \_$th at the end of TF-2, because $L 0_{-} t h$ is the maximum tolerable voltage without test invalidation. Similarly, if $O$ is initialized to $\mathrm{Vdd}$, implying an n-network break, we assume that $O$ will be reduced to L1_th at the end of TF-2. The test becomes invalidated if

$$
C_{O, \text { wiring }} \cdot L 0_{-} t h<\Delta Q_{\text {wiring }} \text { when } O_{\text {init }}=G N D \text {, and }
$$

$C_{O, \text { wiring }} \cdot\left(V d d-L 1_{-} t h\right)<-\Delta Q_{\text {wiring }}$ when $O_{\text {init }}=V d d$

Otherwise, the test is declared to be valid if there are no transient paths to Vdd or GND that will invalidate the test.

The following equations, 3.3 through 3.7 , are taken from Sheu, Hsu, and Ko [18] to express the charge stored on a transistor gate, denoted by $Q_{g}$, and the charge stored by the source and the drain terminals in the channel of a transistor, denoted by $Q_{d}$ and $Q_{s}$. Additionally, we included the sensitivity of model parameters to transistor lengths and widths. These equations are for an nMOS transistor. For a pMOS transistor, the right hand sides of Equations 3.3 to 3.7 need to be negated together with the interterminal voltages.
Subthreshold region, $V_{g s} \leq V_{t h}$ and $V_{g b}>z v f b$ :

$$
\begin{gathered}
Q_{g}=\frac{c a p \cdot z k 1^{2}}{2} \cdot\left(-1+\sqrt{\left.1+\frac{4 \cdot\left(V_{g b}-z v f b\right)}{z k 1^{2}}\right)}\right. \\
Q_{d}=Q_{s}=0
\end{gathered}
$$

Triode region, $V_{g s}>V_{t h}$ and $V_{d s} \leq V_{D S A T}$ :

$$
\begin{gathered}
Q_{g}=\operatorname{cap} \cdot\left(V_{g s}-z v f b-z p h i\right) \quad \text { with } V_{d s}=0 \\
Q_{d}=Q_{s}=-0.5 \cdot \operatorname{cap} \cdot\left(V_{g s}-V_{t h}\right) \quad \text { with } V_{d s}=0
\end{gathered}
$$

Saturation region, $V_{g s}>V_{t h}$ and $V_{d s}>V_{D S A T}$ :

$$
Q_{g}=\operatorname{cap} \cdot\left(V_{g s}-z v f b-z p h i-\left(V_{g s}-V_{t h}\right) /\left(3 \cdot \alpha_{x}\right)\right)
$$

A term that starts with " $\mathrm{z}$ " in the equations above such as $z v f b$ or $z p h i$ is a BSIM electrical parameter taking the transistor width and length into account [13]. cap $=C_{o x}$. $(W-D W) \cdot(L-D L)$ where $C_{o x}$ is the gate-oxide capacitance per unit area, $W$ and $L$ are the drawn transistor width and length, $D W$ and $D L$ are the changes to $W$ and $L$ due to various fabrication steps. For the definitions of $V_{t h}, \alpha_{x}$, and $V_{D S A T}$, and for the reason we assumed $V_{d s}$ to be zero in Equations 3.5 and 3.6, please see our technical report [10].

To compute $\Delta Q_{g, f}$ in Equation 3.1 we use Equations 3.3, 3.5 , and 3.7 depending on the region the fanout transistor $f$ is in. To compute $\Delta Q_{d s, t}$ in Equation 3.2 we use Equations 3.4 and 3.6 depending again on the region transistor $t$ is in. We also include in $\Delta Q_{g, f}$ and $\Delta Q_{d s, t}$ the charge difference due to the gate-diffusion overlap capacitances.

The reverse biased $\mathrm{p}-\mathrm{n}$ junction between the diffusion region and the bulk of a transistor forms the capacitance $C_{p-n}$, whose expression is given in Massobrio and Antognetti [13] as a function of the reverse bias voltage $V_{r}$. Integrating $C_{p-n}$ from $V_{r, \text { init }}$ to $V_{r, \text { final }}$, we obtain the following charge expression for the $\mathrm{p}-\mathrm{n}$ junction.

$$
\begin{aligned}
\Delta Q_{p-n}= & \left.\frac{C_{j s w} \cdot P_{d i f f} \cdot \phi_{j}}{1-m_{j s w}} \cdot\left(1+\frac{V_{r}}{\phi_{j}}\right)^{\left(1-m_{j s w}\right)}\right|_{V_{r, \text { init }}} ^{V_{r, f i n a l}}+ \\
& \left.\frac{C_{j} \cdot A_{d i f f} \cdot \phi_{j}}{1-m_{j}} \cdot\left(1+\frac{V_{r}}{\phi_{j}}\right)^{\left(1-m_{j}\right)}\right|_{V_{r, \text { init }}} ^{V_{r, f i n a l}}
\end{aligned}
$$

where $A_{\text {diff }}$ and $P_{\text {diff }}$ denote the area and the perimeter of the diffusion, and the other new parameters are determined by the fabrication process used. The $\Delta Q_{p-n}$, fen term in Equation 3.2 is computed using Equation 3.8 for node $f \mathrm{cn}$.

\subsection{Initial and Final Voltages}

In this section, we describe how we determine the worst case voltages at transistor terminals at $t_{\text {init }}$ and at $t_{\text {final }}$ in order to compute $\Delta Q_{\text {wiring }}$ in Equation 3.1. We use only six voltage levels, which are Vdd, GND, L0_th, L1_th, max_n, and min_p, where max_n is the maximum voltage an n-network internal node can achieve through a path to Vdd without any Miller feedthrough effect, and min_p is the minimum voltage a p-network internal node can achieve through a path to GND without any Miller feedthrough effect. For the $1.2 \mu$ process we used, max_n was around $3.3 \mathrm{~V}$, and min_p was around $1.2 \mathrm{~V}$ with $\mathrm{Vdd}$ equal to $5 \mathrm{~V}$. 
In order to compute $\Delta Q_{d s, t}$ and $\Delta Q_{p-n, f e n}$ in Equation 3.2 , we need the gate voltages at $t_{\text {init }}$ and at $t_{\text {final }}$ for every transistor $t$ connected to $f c n$, which we denote as $\mathbf{V}_{\mathbf{g}, \mathbf{t}, \mathbf{i n i t}}$ and $\mathbf{V}_{\mathbf{g}, \mathbf{t} \text {,final }}$, and we need the initial and final voltages of $f c n$, which we denote as $\mathbf{V}_{\text {fcn,init }}$ and $\mathbf{V}_{\text {fcn,final. Let us }}$ assume that node $f c n$ is an internal node in the faulty cell, and not the output node. There are two cases to consider:

CASE 1 : There is at least one path of transistors from fcn to $O$ such that the gates of all these transistors are $\mathrm{S} 0$ if $f c n$ is in the p-network, and S1 if $f c n$ is in the $\mathrm{n}$ network. Under this, there are four subcases depending on whether $f \mathrm{cn}$ is in the p-network or in the n-network, and whether $O$ is initialized to GND (p-network break) or Vdd (n-network break). Due to lack of space, we only discuss the two subcases where $f c n$ is in the n-network. The other two subcases where $f c n$ is in the p-network are similar.

Subcase 1.1 : Node $f c n$ is in the n-network, and $O$ is initialized to GND. In this case, $V_{f e n, i n i t}=G N D$, and $V_{\text {fen, final }}=L_{0} t h$. Table 2 shows how the worst case $V_{g, t, i n i t}$ and $V_{g, t, f i n a l}$ values are determined depending on the logic value at $t$ 's gate $\mathbf{g t}$.

\begin{tabular}{|c|c|c|}
\hline Logic value at $g t$ & $V_{g, t, \text { init }}$ & $V_{g, t, \text { final }}$ \\
\hline \hline $01,11,0 \mathrm{X}, \mathrm{X} 1, \mathrm{XX}, 1 \mathrm{X}$ & GND & Vdd \\
\hline $\mathrm{S} 0,00,10, \mathrm{X} 0$ & GND & GND \\
\hline $\mathrm{S} 1$ & Vdd & Vdd \\
\hline
\end{tabular}

Table 2: Worst case gate voltages for Subcase 1.1

The non-obvious cases in Table 2 are when the logic values at $g t$ are 11 and 10 . When the logic value is 11 , it is possible due to a glitch that the voltage at $g t$ is GND at $t_{\text {init }}$. Even when the voltage of $g t$ at $t_{\text {init }}$ is $\mathrm{Vdd}$, the following scenario might occur after $t_{\text {init }}$ : While $O$ is at GND voltage, a glitch causes a falling transition at $g t$, which forces the voltage at fen go below GND, which makes the $\mathrm{p}-\mathrm{n}$ junction between $f c n$ and the bulk of $t$ forward-biased, because the bulk of an nMOS transistor is connected to GND. This way, positive charge is transferred from $t$ 's bulk to node fcn. Note that this charge transfer is happening during the floating period, which will violate our charge conservation assumption of Section 3.1 during the floating period. So, by assuming $V_{g, t, \text { init }}$ to be GND, we are effectively moving the beginning of the floating period from $t_{\text {init }}$ to the point this charge transfer is completed, this way we can still assume charge conservation. The reason we take $V_{g, t \text {, init }}$ to be GND when the logic value at $g t$ is 10 is the same.

In the following cases and subcases, we will omit the reasoning behind our decisions due to lack of space. Our technical report [10] explains the reasons for these decisions.

Subcase 1.2 : $f c n$ is in the n-network, and $O$ is initialized to $\mathrm{Vdd}$. In this case, $V_{f c n, i n i t}=\max n$. Assume max $n \geq L 1 \_t h$, then $V_{f c n, f i n a l}=L 1_{-} t h$, and Table 3 shows how the worst case $V_{g, t, i n i t}$ and $V_{g, t, f i n a l}$ values are determined for transistor $t$ connected to node $f \mathrm{cn}$. The case for max_n<L1_th is described in our technical report [10].

CASE 2 : The condition for CASE 1 is not satisfied, and there is no path of transistors from $f \mathrm{cn}$ to $O$ such that

\begin{tabular}{|c|c|c|}
\hline Logic value at $g t$ & $V_{g, t, \text { init }}$ & $V_{g, t, \text { final }}$ \\
\hline \hline $10,1 \mathrm{X}, \mathrm{X} 0, \mathrm{XX}$ & Vdd & GND \\
\hline $\mathrm{S} 0,00,0 \mathrm{X}$ & GND & GND \\
\hline $\mathrm{S} 1,11, \mathrm{X} 1$ & Vdd & Vdd \\
\hline 01 & GND & Vdd \\
\hline
\end{tabular}

Table 3: The worst case gate initial and final voltages for Subcase 1.2, $\max _{-} n \geq L 1 \_t h$

the gates of all these transistors are $\mathrm{S} 1$ if $f \mathrm{cn}$ is in the $\mathrm{p}$ network, and S0 if $f c n$ is in the n-network. This case is for intermittent connections between $f c n$ and $O$ during the floating period. As in CASE 1, there are again four subcases. Due to lack of space, we only discuss two subcases where $f$ cn is in the n-network. The other two subcases are similar.

Subcase 2.1 : $f c n$ is in the n-network, and $O$ is initialized to GND. In this case, if $f c n$ is connected to GND at the end of TF-1, then $V_{\text {fen init }}=G N D$, otherwise $V_{f e n, i n i t}=m a x_{-} n$. If $f c n$ is connected to $O$ at the end of TF-2, then $V_{f c n, f i n a l}=L 0 \_t h$, otherwise $V_{f c n, f i n a l}=G N D$.

For any transistor $t$ connected to $f c n$, if the logic value at $t$ 's gate $g t$ is $\mathrm{S} 0$ or $\mathrm{S} 1$, then the initial and final $g t$ voltages are both GND or both Vdd, respectively. Otherwise, we take the initial voltage as GND, and the final voltage as Vdd.

Subcase 2.2 : $f c n$ is in the n-network, and $O$ is initialized to Vdd. If $f c n$ is connected to $O$ at the end of TF-1, then $V_{f e n, i n i t}=\max x_{-} n$, otherwise $V_{f e n, i n i t}=G N D$. If $f c n$ is connected to $O$ at the end of TF-2, and $L 1_{-} t h<\max x_{-} n$, then $V_{f c n, f i n a l}=L 1 \_t h$, otherwise $V_{f c n, f i n a l}=\max n$.

For any transistor $t$ connected to $f \mathrm{cn}$, if the logic value at $t$ 's gate $g t$ is neither $\mathrm{S} 0$ nor $\mathrm{S} 1$, then we take the initial voltage for $g t$ as $\mathrm{Vdd}$, and the final voltage as GND. When $g t$ 's logic value is $\mathrm{S} 0$ or $\mathrm{S} 1$, then the initial and final $g t$ voltages are both GND or both Vdd, respectively. This completes Subcase 2.2.

When $f c n$ is the same node as $O$, and $O$ is initialized to GND, we determine the initial and final gate voltages of all the transistors, either in the n-network or p-network, connected to $O$ as shown in Table 2. Obviously, $V_{f e n, i n i t}=$ $G N D$ and $V_{f e n, f i n a l}=L 0 \_t h$ in this case. The case when $O$ is initialized to $\mathrm{Vdd}$ is similar.

In order to estimate the worst case Miller feedback effects, we need to compute $\Delta Q_{g} f$ in Equation 3.1 for each fanout transistor $f$ of $O$. For this, the initial and final voltages at all the terminals of $f$ are needed. There are four cases depending on whether $f$ is an nMOS or a pMOS transistor, and whether $O$ is initialized to GND or Vdd. Due to lack of space, we only discuss the two cases where $f$ is an nMOS transistor. The other two cases are similar.

Let $\mathbf{V}_{\mathbf{g}, \mathbf{f}, \text { init }}$ and $\mathbf{V}_{\mathbf{g}, \mathbf{f}, \text { final }}$ denote the initial and final voltages at $f$ 's gate. Obviously, $V_{g, f \text {,init }}=G N D$ and $V_{g, f, f i n a l}=L 0_{-} t h$ when $O$ is initialized to GND, and $V_{g, f, i n i t}=V d d$ and $V_{g, f, f i n a l}=L 1_{\_}$th when $O$ is initialized to Vdd. Let ds denote the drain or the source terminal of $f$. Let us assume that $d s$ is an internal node, that is, it is neither GND nor the output of cell f $\mathbf{c}$ in which $f$ is located, then routines GetNodeInitFinal and Get_MFB_InitFinal in Figure 3 show how we determine the initial and final voltages 


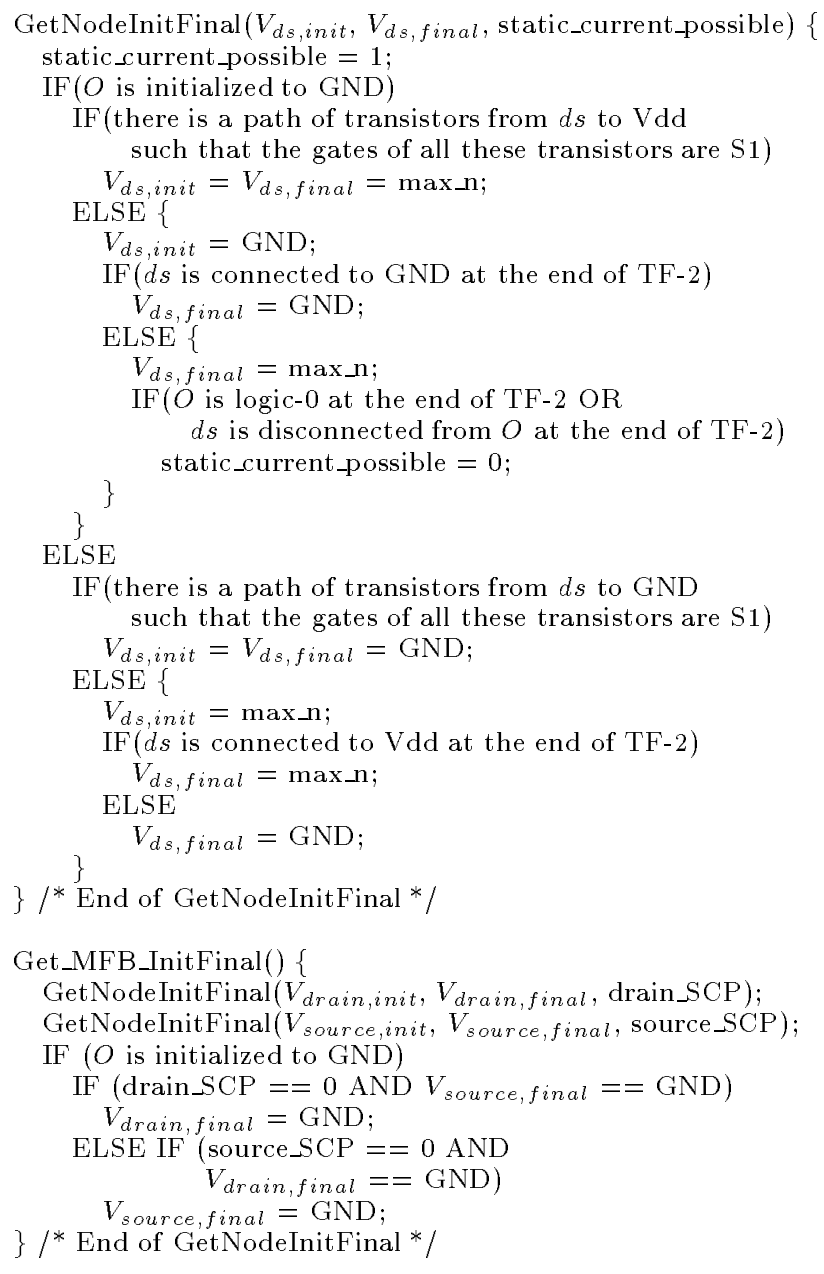

Figure 3: Determining drain/source initial/final voltages for the Miller feedback effect

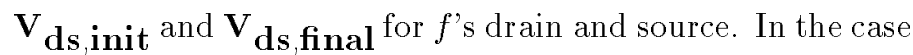
$O$ is initialized to $G N D$, when $O$ reaches L0_th at the end of TF-2, the nMOS transistor $f$ will be weakly turned on. If the output of $f c$ is sensitized to $O$, then a static current will be flowing in $f c$. The flag static_current_possible in routine GetNodeInitFinal is used to determine when it is impossible for $f c$ 's output to be sensitized to $O$ due to the logic values at the side-inputs of $f c$. When $d s$ is $f c$ 's output, then the max_n terms in Figure 3 will be replaced by Vdd.

\section{IMPLEMENTATION AND RESULTS}

We implemented the fault simulation algorithm described in the previous section. We obtained the BSIM model parameters from MOSIS for the $1.2 \mu$ Orbit n-well fabrication process. We extracted the wiring capacitances using Magic. We took L0_th to be $1.8 \mathrm{~V}$ and L1_th to be $3.2 \mathrm{~V}$.

For every MCNC standard cell used in the ISCAS85 circuits, we used the public domain ext2spice program to determine the area and the perimeter of the diffusion region for the drain and source terminals of each transistor in the cell. We used an inductive fault analysis tool, Carafe [8, 17], to get a list of realistic network breaks in the cell.

For each internal node in each faulty cell, our program generates the connection function between the internal node and the faulty cell output, where the connection function between two nodes in a cell denotes a sum-of-products expression, where each product term describes the condition to activate a transistor path between the two nodes, and a product term exists for every possible transistor path between the two nodes. Please see our technical report [10] for other connection functions we generate.

The standard cells are processed as described above only once, not every time a circuit is fault simulated. Our program performs parallel pattern simulation using our elevenvalue logic algebra to determine the logic value on each wire in time frames 1 and 2 in the fault-free circuit. Then, we perform parallel pattern single fault propagation [4] only in TF2 to determine the stuck-at-0 and stuck-at- 1 detectability of the wires. If a stuck-at- 0 on a wire is detectable in TF- 2 and the wire is logic-0 in TF-1, then our program checks for possible transient paths to $\mathrm{Vdd}$ and computes the $\Delta Q_{\text {wiring }}$ in Equation 3.1 for the p-network breaks in the cell that drives the wire. The n-network breaks are processed similarly.

We ran our simulator on a DECstation 5000/240 with $128 \mathrm{Mb}$ of memory. In the experiments shown in Table 4, we kept generating random patterns until a certain number of successive random patterns do not detect any further network break, and that number is proportional to the number of cells used in the circuit. We call a wire in a circuit short wire if its capacitance to GND is less than or equal to $35 \mathrm{fF}$. We chose $35 \mathrm{fF}$ arbitrarily mostly because the wiring capacitance we used in Figure 1 was also $35 \mathrm{fF}$. All circuits but c1355 and c6288 have double digit short wire percentages, because all these circuits have XOR or XNOR gates in them, and such a gate consists of two primitive gates with about $10 \mathrm{fF}$ wiring between them. Note that it is easier for a test to be invalidated by Miller effects and charge sharing as the wiring capacitance gets smaller. The last two columns give the percentages of network breaks (NBs) covered by random vectors and uncompacted single-stuckat (SSA) test sets, respectively. The low coverage by SSA vectors hint a need for test generation for network breaks.

Because we use only six voltage levels for our charge difference computations, a look-up table can be constructed for all combinations of these voltages in the charge Equations 3.3 to 3.8 . We constructed such a look-up table only for the $\left(1+V_{r} / \phi_{j}\right)^{\left(1-m_{j}\right)}$ and $\left(1+V_{r} / \phi_{j}\right)^{\left(1-m_{j s w}\right)}$ terms in Equation 3.8, since taking the power of a real number is computationally expensive. Even though we did not construct look-up tables for other equations, we ended up with reasonable CPU times as shown in Table 4, in fact, our CPU times per vector are always better than the ones by Di and Jess [3], where they used an HP-9000/700.

Table 5 shows our fault coverage results using 1024 random vectors for each circuit. The fault coverage numbers in an "SH off" column are obtained by turning the static hazard identification off, that is, every 00 is treated as S0, and every 11 is treated as $\mathrm{S} 1$. In an "SH on" column, static hazard identification is on. In Table 5, "charge off" means that 


\begin{tabular}{|c|c|c|c|c|c||c|}
\hline Ct. & $\begin{array}{c}\text { \# of } \\
\text { NBs }\end{array}$ & $\begin{array}{c}\text { \% of } \\
\text { short } \\
\text { wires }\end{array}$ & $\begin{array}{c}\text { \# of } \\
\text { random } \\
\text { vecs }\end{array}$ & $\begin{array}{c}\text { CPU } \\
\text { per vec } \\
(\mathrm{ms} .)\end{array}$ & $\begin{array}{c}\text { FC } \\
(\%)\end{array}$ & $\begin{array}{c}\text { FC (\%) } \\
\text { with } \\
\text { SSA vecs }\end{array}$ \\
\hline $\mathrm{c} 432$ & 931 & 27.7 & 4000 & 3.8 & 87.8 & 59.0 \\
\hline c499 & 1403 & 44.0 & 5856 & 7.3 & 63.4 & 56.8 \\
\hline c880 & 1337 & 20.6 & 7360 & 2.0 & 94.8 & 76.7 \\
\hline c1355 & 2174 & 4.9 & 9120 & 9.4 & 74.5 & 61.2 \\
\hline c1908 & 2235 & 34.0 & 22528 & 9.0 & 75.5 & 57.8 \\
\hline c2670 & 3427 & 16.7 & 17920 & 6.2 & 78.2 & 69.5 \\
\hline$c 3540$ & 4947 & 17.0 & 29984 & 13.1 & 91.6 & 67.0 \\
\hline c5315 & 7607 & 20.3 & 70528 & 15.1 & 94.0 & 73.6 \\
\hline$c 6288$ & 10760 & 7.9 & 138624 & 128.2 & 87.4 & 61.5 \\
\hline$c 7552$ & 9955 & 23.2 & 90912 & 22.3 & 86.5 & 70.6 \\
\hline
\end{tabular}

Table 4: Results using random and SSA vectors

\begin{tabular}{|c|c|c|c|c|c|}
\hline \multirow[b]{2}{*}{ Circuit } & \multirow[b]{2}{*}{ SH on } & \multirow[b]{2}{*}{ SH off } & \multicolumn{2}{|c|}{ charge off } & \multirow{2}{*}{$\begin{array}{l}\text { charge off } \\
\text { paths off }\end{array}$} \\
\hline & & & SH on & SH off & \\
\hline c432 & 84.0 & 89.5 & 88.0 & 92.6 & 98.7 \\
\hline c499 & 60.4 & 80.8 & 73.0 & 90.1 & 99.5 \\
\hline$c 880$ & 89.3 & 90.6 & 92.4 & 93.3 & 98.6 \\
\hline c1355 & 69.6 & 83.3 & 77.6 & 87.8 & 96.9 \\
\hline c1908 & 54.8 & 63.5 & 63.6 & 70.9 & 86.5 \\
\hline c2670 & 71.2 & 76.5 & 75.1 & 79.6 & 85.7 \\
\hline c3540 & 77.1 & 85.6 & 81.7 & 88.7 & 96.6 \\
\hline c5315 & 83.7 & 91.0 & 87.6 & 93.9 & 98.9 \\
\hline c6288 & 76.8 & 96.0 & 82.8 & 97.2 & 99.9 \\
\hline c7552 & 72.0 & 80.7 & 76.9 & 84.4 & 89.9 \\
\hline
\end{tabular}

Table 5: Fault coverage results using 1024 random patterns with varying accuracy levels

the computation of $\Delta Q_{\text {wiring }}$ is turned off, that is, Miller effects and charge sharing are ignored. The "paths off" term means that transient paths to Vdd or GND are ignored. Note that when all of Miller effects, charge sharing, and transient paths are ignored, detection of a network break is only determined by SSA detection in TF-2 and the value of the cell output in TF-1, so static hazards have no relevance. A fault coverage value in this last column might be greater than the SSA coverage of the circuit. For instance, the coverage value for c6288 in the last column is $99.9 \%$ while the SSA coverage for this circuit is $99.4 \%$, because most of the undetectable SSA faults in c6288 are on fanout branches, and SSA detectability of fanout branches are not relevant in network break detection.

Comparing the "SH on" with the "SH off" columns shows that static hazard identification makes a quite significant difference. Another observation is that disabling Miller effects and charge sharing has a significant impact on fault coverage even though the impact of disabling transient path identification is bigger.

\section{CONCLUSION}

The main conclusions from our results have been that Miller effects and charge sharing are significant enough not to be ignored, transient paths are the main cause of test invalidation, static hazard identification is very important, and test generation for network breaks may be necessary to achieve high fault coverage.

\section{Acknowledgments}

We thank Don Speck for stimulating discussions on VLSI, Alvin Jee for his help with Carafe, and Andy Burstein for his help with the ext2spice software.

\section{References}

[1] V.H. Champac, A. Rubio, and J. Figueras. Electrical model of the floating gate defect in CMOS IC's: Implications on IDDQ testing. IEEE Transactions on CAD, March 1994.

[2] H. Cox and J. Rajski. Stuck-open and transition fault testing in CMOS complex gates. ITC, 1988.

[3] C. Di and J.A.G. Jess. On accurate modeling and efficient simulation of CMOS opens. ITC, 1993.

[4] J.A. Waicukauski et al. Fault simulation for structured VLSI. VLSI Systems Design, Dec. 1985.

[5] Z. Barzilai et al. Efficient fault simulation of CMOS circuits with accurate models. ITC, 1986.

[6] M. Favalli, M. Dalpasso, P. Olivo, and B. Ricco. Modeling of broken connections faults in CMOS ICs. European Design and Test Conference, 1994.

[7] C.F. Hawkins, J.M. Soden, A.W. Righter, and F.J. Ferguson. Defect classes - an overdue paradigm for CMOS IC testing. ITC, 1994.

[8] A. Jee and F. J. Ferguson. Carafe: An inductive fault analysis tool for CMOS VLSI circuits. VLSI Test Symposium, 1993.

[9] N.K. Jha and J.A. Abraham. Design of testable CMOS logic circuits under arbitrary delays. IEEE Transactions on CAD, July 1985.

[10] H. Konuk and F. J. Ferguson. Accurate and efficient fault simulation of realistic CMOS network breaks. Technical Report UCSC-CRL-94-40, Comp. Eng. Dept., UC Santa Cruz. URL:ftp://ftp.cse.ucsc.edu/pub/tr/1994/ucsc-crl-9440.

[11] K.-J. Lee and M.A. Breuer. On the charge sharing problem in CMOS stuck-open fault testing. ITC, 1990.

[12] W.M. Maly, P.K. Nag, and P. Nigh. Testing oriented analysis of CMOS ICs with opens. ICCAD, 1988.

[13] G. Massobrio and P. Antognetti. Semiconductor Device Modeling with SPICE. McGraw-Hill, 1993.

[14] Meta-Software. HSPICE User's Manual: Elements and Models. 1992.

[15] S.M. Reddy, M.K. Reddy, and J.G. Kuhl. On testable design for CMOS logic circuits. ITC, 1983.

[16] M. Renovell and G. Cambon. Electrical analysis and modeling of floating-gate fault. IEEE Transactions on CAD, Nov. 1992 .

[17] J. Rogenski and F. J. Ferguson. Characterization of opens in logic circuits. IEEE ASIC Conference, 1994.

[18] B.J. Sheu, W.-J. Hsu, and P.K. Ko. An MOS transistor charge model for VLSI design. IEEE Transactions on CAD, April 1988.

[19] B. Underwood, S. Kang, W.-O. Law, and H. Konuk. Fastpath: Robust path-delay test generator for standard scan designs. ITC, 1994.

[20] J.-F. Wang, T.-Y. Kuo, and J.-Y. Lee. A new approach to derive robust tests for stuck-open faults in CMOS combinational logic circuits. $D A C, 1989$. 